\title{
Tuberculosis in children undergoing hemodialysis
}

This article was published in the following Dove Press journal: International Journal of Nephrology and Renovascular Disease 2I April 2010

Number of times this article has been viewed

\section{Gargah Tahar' \\ Goucha-Louzir Rim² Lakhoua Mohamed Rachid' \\ 'Department of Pediatric Nephrology, ${ }^{2}$ Department of Nephrology, Charles Nicolle Hospital, Tunis, Tunisia}

Correspondence: Gargah Tahar Boulevard 9 Avril, Charles Nicolle hospital,Tunis, Tunisia 1009

Tel +2162306 I5 I5

Email kitomora@yahoo.fr

\begin{abstract}
Tuberculosis (TB) remains a public health problem in Tunisia. Its incidence is higher in immunocompromised hosts than in the general population. In children and during hemodialysis, TB is characterized by the frequency of extrapulmonary localizations and diagnostic difficulties. The aim of this retrospective study is to evaluate the incidence of TB in Tunisian children undergoing hemodialysis and to determine its clinical features as well as the results of chemotherapy.
\end{abstract}

Method: This retrospective study includes seven TB children among 112 children on hemodialysis at the pediatric nephrology department in Charles Nicolle Hospital from 2002 to 2008. The diagnosis of TB was established by a combination of clinical, radiological, biochemical, microbiological, and histological examinations. Treatment with anti-TB drugs, the results of therapy, and the outcome of patients were noted.

Results: There were four girls and three boys aged 10 to 16 years (mean, 13 years). They had been on hemodialysis for 2 to 5 years (mean, 3 years). Noted clinical features were weight loss and fever in five cases, chest pain in one case, cervical lymph node in one case, and spinal pain in one case. The organ systems involved were pleural in two cases, pulmonary in one case, peritoneal in one case, cervical lymphatic in one case, and spinal in one case. One patient was treated empirically with a good response. Diagnosis was made by isolation of mycobacterium TB in three cases, by specific histological signs observed in a lymph node biopsy in one case, in peritoneal biopsy in one case, and in discovertebral biopsy in one case. In the remaining patient, the clinical and radiological presentations were compatible with pulmonary TB. All patients received four anti-TB drugs: isoniazid, rifampicin, pyrazinamide, and ethambutol. One patient died with miliary TB. The other patients had favorable outcomes.

Conclusions: TB in hemodialysis children has a nonspecific clinical presentation. Extrapulmonary locations are most common. Diagnosis is often difficult, but successful outcomes are possible when made at an early stage.

Keywords: child, hemodialysis, tuberculosis

\section{Introduction}

The incidence of tuberculosis (TB) decreased in recent years in Tunisia due to effective vaccination. The annual incidence is estimated at 3.5 per 100,000 children. ${ }^{1}$ Immunocompromised patients, such as those with end-stage renal failure requiring hemodialysis, have an increased risk of developing TB infection. This risk has been estimated as nearly 10- to 25-fold higher among hemodialysis patients than among the general population. ${ }^{2}$ We report the incidence of TB in normal children as well as in hemodialysis children. We discuss the clinical characteristics, diagnostic procedures, and clinical outcomes for TB children on hemodialysis. 


\section{Patients and methods}

We retrospectively analyzed all hemodialysis patients aged less than 18 years in whom the diagnosis of TB was presumptive or confirmed from January 2002 to December 2008. One hundred twelve patients were enrolled. Seven patients were diagnosed with TB. Of these, three cases were considered presumptive, three patients were noted to have positive mycobacterium TB culture, and the other was empirically treated for TB with a good response. The following data were collected for each patient: epidemiological characteristics, risk factors, duration of hemodialysis, clinical symptoms, diagnostic methods, treatment, and TB evolution.

\section{Results}

Among 112 children treated with hemodialysis during the study period, we noted seven cases (25\%) of TB disease: four females and three males with an average age of 13 years (range, 10-16 years). Their duration on hemodialysis ranged from 2 to 5 years (mean, 3 years). The presenting clinical features were weight loss and fever in five cases, chest pain in one case, spinal pain in one case, and cervical lymphadenopathy in one case. Tuberculin skin test was negative in all patients. Chest $X$-ray showed an abundant pleural effusion in two cases and a miliary TB in one case. In these three patients, sputum samples were smear-negative. The fluid from patients with pleurisy showed exudates rich in lymphocyte (95\%). Acid-fast smears using Zeihl-Neelsen acid fast stain and culture in Lowenstein-Jensen medium were performed but proved to be negative. Caseous granulomas were found in pleural biopsy in our patients with TB pleurisy. The culture of tissue biopsy specimens revealed mycobacterium TB in both patients. In patients with peritoneal TB, the diagnosis was established by data from the peritoneal biopsy done by laparoscopy (caseous granulomas).

In the case of miliary TB, sputum culture was positive. In another patient, the clinical and radiological presentations were compatible with pulmonary TB. Noted improvement after anti-TB therapy confirmed this diagnosis. The clinical characteristics of our patients are illustrated in Table 1.

Four drugs, isoniazid (INH), rifampicin (RIF), pyrazinamide (PZA), and ethambutol (EMB) were administered orally to six patients initially for three months followed by only INH and RIF for a further six months to complete a ninemonth therapy. Drugs were administered on dialysis days following the session of dialysis (three days per week). The outcome was favorable. In one patient with miliary TB, INH and RIF was used intravenously. He died at the beginning

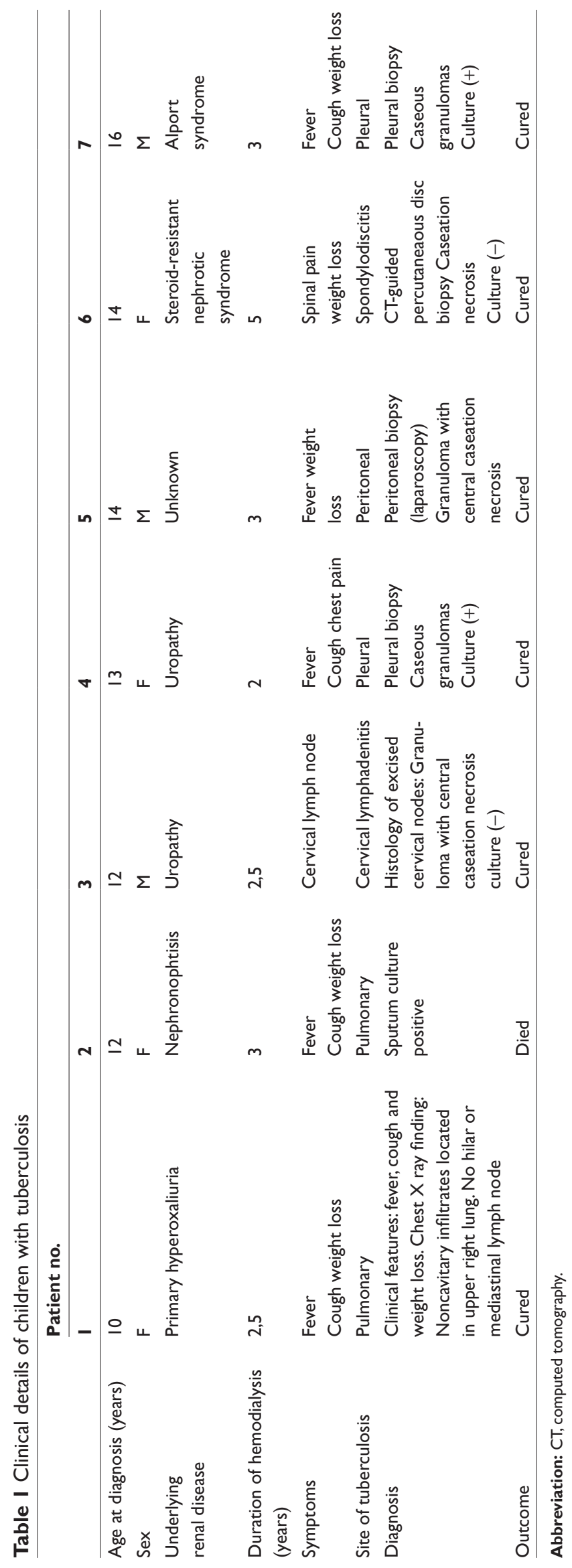


of treatment. TB was considered as the direct cause of death for this patient.

\section{Discussion}

Uremic adults are more susceptible to TB infection due to pathological changes in the airways and impairment in cellular immunity. ${ }^{3}$ Dialysis increases these immunological impairments through depletion of useful proteins, trace metals, and vitamins; it also induces an immune response through biocompatibility of dialysis membrane and dialysis fluid or entry of microorganisms. ${ }^{4}$ Few data are available for uremic children. To our knowledge, nothing has been published in the English literature on TB in hemodialysis children. However, the most recent publication about the child on dialysis reported four cases of TB in children undergoing continuous ambulatory peritoneal dialysis in Turkey. ${ }^{5}$ According to this study, the incidence of TB in children on dialysis is much lower than in adults on dialysis (23.6\% versus $5.7 \%$ ). This has been attributed to the vaccination program which begins at the age of two months and repeated at the age of seven and 12 years. In Tunisia, the vaccination schedule is similar to Turkey apart from the first dose being given immediately at birth then improving immunization at an early age and before the installation of chronic renal disease.

The emergence of TB infection in uremic adults is observed during the early years of hemodialysis. During this phase, the cell-mediated immunity is compromised and promotes infection, especially TB. ${ }^{6}$ Mesiha and colleagues ${ }^{5}$ studied children undergoing peritoneal dialysis, and noted that the diagnosis of TB was made during the first year of dialysis in three patients and before dialysis in another patient. In our study, TB infection took place 24 months after beginning hemodialysis.

The mode of contamination is probably a reactivation of a pre-existing focus but in our study, the TB may be due to an airborne spread in the hospital community with respect to the interval between the beginning of dialysis and diagnosis of TB.

Clinical presentation of TB may be insidious and nonspecific, mimicking uremic symptoms. There are clinical situations that alert us to the presence of TB such as unexplained fever, anorexia, deterioration of nutritional heath, and spinal pain which is often associated to renal osteodystrophy.

The diagnosis of TB is difficult to establish in the case of renal failure by simply using traditional tests. The tuberculin skin test is often negative. Bacteriological proof is rarely obtained by traditional tests such as Zeihl-Neelsen staining, culture, or polymerase chain reaction. ${ }^{7}$ Thus, new, rapid, and it is imperative to utilize accurate diagnostic methods such as blood test measuring the interferon- $\gamma$ released by stimulated T cells. ${ }^{8}$ In our study, and because of the unavailability of these modern methods, invasive diagnostic procedures were performed to prove the TB infection: pleural biopsy, peritoneal biopsy, and percutaneaous disc biopsy. Histopathological examination and culture of tissue obtained by biopsy identified the mycobacterium TB in two cases.

TB has been described in uremic patients along with an infection with predominantly extra pulmonary involvement. ${ }^{9}$ During dialysis, pulmonary TB with cavities was minor whereas extrapulmonary TB and miliary TB were major. ${ }^{10}$ Our data confirm these findings since we found only two patients with pulmonary TB among seven cases.

Apart from the diagnostic difficulties, TB in patients undergoing hemodialysis have a real therapeutic problem. The bioavailability of most drugs are altered by renal failure and hemodialysis. The half-life of INH is prolonged in endstage renal disease. ${ }^{11}$ Consequently, some researchers ${ }^{12}$ use INH in half doses $(5 \mathrm{mg} / \mathrm{kg})$ per day. However, others prefer to use the full dose.

In our study, we prescribe a full dose with good tolerance even in slow acetylates. We recommend giving the dose of INH on dialysis days after the dialysis session. The neurological side effects of INH are preventable by adding pyridoxine in doses of $50 \mathrm{mg}$ per day (half of the recommended adult dose).

The usual dose of RIF is recommended because its metabolism does not depend on renal function. ${ }^{13}$ EMB is usually given in a dose of $20 \mathrm{mg} / \mathrm{kg}$ three times a week after each hemodialysis session. In the same way, we can prescribe the PZA at a dose of 20 to $25 \mathrm{mg} / \mathrm{kg} .{ }^{13} \mathrm{In}$ children on dialysis, the duration of treatment in accordance with new recommendations is 12 months (with INH and RIF and with EMB and PZA added for the first two months). ${ }^{5} \mathrm{We}$ strongly recommend this protocol to avoid the resistance and resurgence of TB after kidney transplantation. The efficiency of this protocol is only possible if the treatment is well monitored and tolerated. ${ }^{14}$ In our study, the outcome was fatal for a patient with miliary TB despite intravenous INH and RIF.

\section{Conclusion}

Dialysis patients and especially children are at high risk of TB. Physicians caring for dialysis children must remain vigilant for signs of TB in this vulnerable population. Two basic points emerge throughout our study and literature review: 
1. Diagnostic difficulties of the disease are due to the insidious and nonspecific clinical presentation and difficulties in identifying the mycobacterium TB by conventional methods.

2. The outcome is often favorable when treatment starts at an early stage and the duration is long enough.

\section{Disclosures}

The authors report no conflicts of interest in this work.

\section{References}

1. Epidemiological Bulletin of the Republic of Tunisia for the year 2008.

2. Chou KJ, Fang HC, Bai KJ, Hwang SJ, Yang WC, Chung HM. Tuberculosis in maintenance dialysis patients. Nephron. 2001;88:138-143.

3. Kato S, Chmielewski M, Honda H, et al. Aspects of immune dysfunction in end-stage renal disease. Clin J Am Soc Nephrol. 2008; 3:1526-1533.

4. Cengiz A. Increased incidence of tuberculosis in patient undergoing haemodialysis. Nephron. 1996;73:421-424.

5. Mesiha E, Necmiye T, Sevcan B. Tuberculosis in children undergoing continuous ambulatory peritonel dialysis. Pediatr Nephrol. 1999;13:577-579.
6. Hussein MM, Bakir N, Boujouleh H. Tuberculosis in patients undergoing maintenance dialysis. Nephrol Dial Transplant. 1990;5: 584-587.

7. Passlent L, Khan K, Richardson Kwang J, Dedia H, Gardam M. Detecting latent tuberculosis infection in haemodialysis patients: A head-tohead comparison of the T-SPOT.TB test, tuberculin skin test, and an expert physician panel. Clin J Am Soc Nephrol. 2007;2:68-73.

8. Lee SSJ, Chou KJ, Su IJ, et al. High prevalence of latent tuberculosis infection in patients in end-stage renal disease on hemodialysis: Comparison of QuantiFERON-TB GOLD, ELISPOT, and tuberculin skin test. Infection. 2009;37:96-102.

9. Lund RJ, Koch MJ, Oldemever JB, Meares AJ, Dunlay RW. Extrapulmonary tuberculosis in patients with end stage renal disease: two case reports and a brief review. Int Urol Nephrol. 2000;32:181-183.

10. Hussein MM, Mooij JM, Roujouleh H. Tuberculosis and chronic renal disease. Semin Dial. 2003;16:38.

11. Ellard GA. Chemotherapy of tuberculosis for patients with renal impairment. Nephron. 1993;64:169-181.

12. Siskind MS, Thienemann D, Kirlin L. Isoniazid-induced neurotoxicity in chronic dialysis patient: Report of three cases and a review of the literature. Nephron. 1993;64:303-306.

13. Launay-Vacher V, Izzedine H, Eray G. Pharmacokinetic considerations in the treatment of tuberculosis in patients with renal failure. Clin Pharmacokinet. 2005;44:221-235.

14. Peloquin CA. Therapeutic drug monitoring in the treatment of tuberculosis. Drugs. 2002;62:2169-2183.

\section{Publish your work in this journal}

The International Journal of Nephrology and Renovascular Disease is an international, peer-reviewed open-access journal focusing on the pathophysiology of the kidney and vascular supply. Epidemiology, screening, diagnosis, and treatment interventions are covered as well as basic science, biochemical and immunological studies. The journal welcomes original research, clinical studies, reviews \& evaluations, expert opinion and commentary, case reports and extended reports. The manuscript management system is completely online and includes a very quick and fair peerreview system, which is all easy to use. Visit http://www.dovepress.com/ testimonials.php to read real quotes from published authors. 